\title{
¿Qué hacen los menores en internet? Usos de las TIC, estrategias de supervisión parental y exposición a riesgos
}

\author{
Ana $M^{a}$ Giménez ${ }^{1}$, José A. Luengo ${ }^{2}, M^{a}$ José Bartrina $^{3}$ \\ ${ }^{1}$ Departamento de Educación, Universidad Católica San Antonio de Murcia, \\ Murcia \\ ${ }^{2}$ Facultad de Educación, Universidad Camilo José Cela, Madrid \\ ${ }^{3}$ Departamento de Justicia, Generalitat de Cataluña
}

\section{España}

Correspondencia: Ana $M^{a}$ Giménez Gualdo. Dpto de Educación, Facultad de Ciencias Sociales y de la Comunicación, Universidad Católica San Antonio de Murcia. Campus de los Jerónimos, C.P. 30107, Guadalupe (Murcia), España. E-mail: amgimenez@ucam.edu

(C) University of Almería and Ilustre Colegio Oficial de la Psicología de Andalucía Oriental (Spain) 


\section{Resumen}

Introducción. Las investigaciones actuales insisten en la accesibilidad y consumo que los menores hacen de las redes sociales, chats y WhatsApp. Sin embargo, esta realidad no va asociada a una supervisión parental activa que los proteja de los numerosos riesgos existentes en la red. Este trabajo analiza la percepción de estudiantes de Murcia sobre los usos que hacen del móvil y el ordenador, las estrategias de supervisión de sus progenitores y su relación con experiencias de cyberbullying y uso problemático de las tecnologías.

Método. La muestra estuvo compuesta por 1914 estudiantes con edades comprendidas entre los 11 y 21 años $(M=13.83$, DT = 2.03) de centros públicos y privados de educación primaria y secundaria de la Región de Murcia (España). La selección muestral fue aleatoria por conglomerados. La recogida de información se realizó a partir de una encuesta estructurada autoinformada diseñada ad hoc.

Resultados. El acceso a internet a través del teléfono móvil (70\%) y el ordenador (99\%) está generalizado con un $68.9 \%$ que dicen dedicar entre 1-2 horas/día al móvil y un $67.1 \%$ al ordenador. Sin embargo, destaca que entre un 10\%-15\% digan dedicar más de cuatro horas diarias a ambas tecnologías. Aparecen diferencias significativas por sexo en el consumo del móvil, como también por sexo y nivel educativo en los diferentes usos del teléfono y el ordenador. Para ambas tecnologías impera el uso comunicativo y el social a través del WhatsApp y las Redes Sociales. Los resultados sobre la supervisión parental manifiestan su escasez entre los menores, siendo las estrategias más comunes preguntar y limitar el tiempo de conexión. Destaca la relación significativa entre supervisión parental, experiencias de cyberbullying y conductas relacionadas con el uso problemático de las TIC.

Discusión y conclusiones. La implicación de la familia a modo de supervisición durante la conexión de los menores a las TIC es un importante factor de protección ante los riesgos a los que se exponen los menores como son el cyberbullying y la ciberadicción. Se advierte la necesidad de reforzar la mediación parental para el logro de un uso más responsable de las TIC y evitar riesgos.

Palabras Clave: adolescentes, superisión parental, riesgos en la red, cyberbullying. 


\section{Abstract}

Introduction. Current research highlights the accessibility and use that young people do of social networks, chatrooms and WhatsApp. However, this situation does not connect with active parental mediation that protects them of several risks on internet. This study analyzes Murcian students' perception of mobile phones and computers use, parental mediaton strategies and its relation with cyberbullying and problematic use of technologies.

Method. The sample was 1914 students between 11 to 21 years old $(\mathrm{M}=13.83$, DT = 2.03) from primary and secondary public and private schools from the Region of Murcia (Spain). Participants were randomly and stratified selected. A self-informed structured survey was used to collect information.

Results: Access to internet by mobile phones (70\%) and computers (99\%) is highly widespread with a $68.9 \%$ of students who use mobiles and a $67.1 \%$ who use computer between $1-2$ hours per day. Nevertheless, there was between $10 \%$ and $15 \%$ of students who use technologies more than four hours per day. There were significant differences in the time use of mobiles by sex as well as differences by sex and level on uses for mobile and computers. The most common use for both technologies is for communication and relationship by WhatsApp and social networks. Findings on parental supervison among children show that is limited, being ask questions or limitate time use the most common strategies developed. It is noted that there is a significat relation between parental supervision, cyberbullying and behaviors related with problematic ITC use.

Discussion and Conclusions. Family implication to guidance and supervise young people when are connected to ICT is an important factor to protect them against internet risks like cyberbullying or cyberadicction. We warn about the need to reinforce parental supervision to young people be able to make a more responsible use of ICT and prevent risks.

Keywords: young people, parental supervision, internet risks, cyberbullying. 


\section{Introducción}

El análisis del ciberbullying también conocido como cyberbullying o electronic bullying abarca desde la última década una dimensión mundial (Smith, 2016). La primera aproximación a su estudio data del año 2000 con Finkelhor, Mitchell y Wolak que ya encontraban un $6 \%$ de escolares implicados. Hasta el momento son numerosas las investigaciones que han tratado de indagar en la naturaleza de este acoso, sus causas y el grave impacto emocional y psicológico que conlleva, principalmente para las víctimas (Giménez, Hunter, Durkin, Arnaiz y Maquilón, 2015; Hase, Goldberg, Smith, Stuck y Campain, 2015; Kowalski, Giumetti, Schroeder y Lattaner, 2014; Mishna, McInroy, Lacombe-Duncan y Daciuk, 2015) como también su impacto en el rendimiento académico de los estudiantes (Bellamy y Yousef, 2015). Estudios recientes confirman la relación existente entre el incremento del cyberbullying y el aumento de la muerte infantil por causas no naturales ( $\mathrm{Fu}$, Chan e Ip, 2014).

Por otro lado, datos a nivel mundial dan cuenta de su peligrosa extensión e incremento entre la población adolescente (Lucas, Pérez y Giménez, 2016). En EEUU, Hinduja y Patchin (2015) sitúan la media de cibervictimización en el 34.4\% del que un $21 \%$ afirma ser acosada de forma reiterada y de ciberagresión del $14.6 \%$ con un 5.5\% más frecuente. En Australia, las cifras son menores, aunque giran en torno al $11 \%$ entre escolares varones de 10 a 17 años (Sakellariou, Carroll y Houghton, 2012), resultados similares a los encontrados en Unión Europea con un 12\% (Dalla, Di Pietro, Morel y Psaila, 2016). El último informe de Save The Children (Calmaestra et al., 2016) señala un 9.3\% de bullying y un 6.9\% de cyberbullying con un porcentaje de ciberacoso en la Región de Murcia superior a la media nacional junto a otras comunidades como Andalucía y Melilla. Entre las formas más frecuentes destaca el insulto a través de la telefonía móvil y el ordenador a través de WhatsApp, Messenger y las redes sociales. Son precisamente las redes sociales el uso preferente para la comunicación y otros fines por parte de adolescentes y cada vez más niños, las que se convierten en una herramienta perfecta para ejercer y padecer este tipo de acoso (Del Río, Sádaba y Bringué, 2010). Se ha comprobado también que, ser usuario frecuente de las mismas aumenta las posibilidades de convertirse en ciberagresor y cibervíctima (León, Felipe, Fajardo y Gómez, 2012). Esto es en muchos casos desconocido por parte de las familias que además también ignoran los perfiles que sus hijos tienen en dichas redes y qué material o información comparten y con quién (Cloquell, 2015). 
El uso y consumo cada vez mayor de las TIC por parte de niños y adolescentes los sitúa en un panorama de diversos riesgos entre los que cabe destacar: el acceso a contenidos inapropiados, contacto con desconocidos, grooming, amenazas contra la privacidad, suplantación de la identidad, sexting, el propio cyberbullying, y la problemática cada vez más creciente de la adicción a internet, a los móviles y TIC en general (Carbonell et al., 2012; Fajardo et al., 2013). En España datos del Observatorio Nacional de las Telecomunicaciones (ONTSI), sitúan el porcentaje de acceso a internet por parte de niños y niñas en el $94.6 \%$ y del teléfono móvil en el 69.8\%. Por Comunidades Autónomas, la Región de Murcia se sitúa en el 89\% de menores con acceso a un ordenador y un $73 \%$ de teléfono móvil, en la mayoría de las ocasiones, de uso propio (ONTSI, 2016). Sobre el consumo excesivo de internet, Ochaíta, Espinosa y Gutiérrez (2011) indican conductas de navegación sin rumbo fijo (31\%), la necesidad constante de conexión (12\%) y sentirse disgustados ante la no conexión (16\%) como los principales síntomas mostrados por los jóvenes. Estudios más actuales, señalan que un 13.6\% de adolescentes reconocen un uso problemático de internet y un $2.4 \%$ del teléfono móvil (Muñoz et al., 2016).

Ante esta situación es clave la labor de medicación y supervisión parental para educar y concienciar a los menores en un uso más responsable de las TIC. Esta labor es muy importante para prevenir a los menores de los riesgos en la red que tanto impactan en su contexto personal y social (Navarro y Serna, 2016). En los últimos años se han sucedido estudios que tratan de conocer las estrategias que padres y madres ponen en marcha en su labor de supervisión. La mayor parte de ellos concluye que, gran parte de los escolares afirman no ser supervisados por sus padres durante su acceso a internet como tampoco tener impuestos horarios (Berríos, Buxarrais y Garcés, 2015), mientras que a la par los padres tienden a sobreestimar tal labor (Garmendia, Casado, Martínez y Garitaonandia, 2013). Esto se concreta además en la herramienta de las redes sociales de uso preferente en la comunicación y socialización de los y las adolescentes, donde se encuentra únicamente un $22 \%$ que afirman tener algún tipo de regulación por parte de sus padres cuando las utilizan (Colás, González y de Pablos, 2013).

Entre las formas y estrategias más comunes de supervisión destacan aquellas de tipo restrictivo, instructivo o activas, estrategias de seguimiento, y otras de carácter técnico (Garmendia et al., 2013; Martínez de Morentin y Medrano, 2012). Análisis cualitativos realizados con una muestra de padres ingleses con hijos entre los 7 y 10 años concluyen que, entre las 
estrategias que más afirman desarrollar destacan la supervisión técnica por la que ligan la cuenta de Facebook del hijo/a a la propia, poner restricciones en el ordenador o chequear el móvil (Monks, Mahdavi y Rix, 2016). Esto coincide con los resultados encontrados en el informe EU Kids Online, donde son mayoría los padres afirman ejercer una mediación de tipo activo y restrictivo frente a actitudes más pasivas (Helsper, Kalmus, Hasebrink, Sagvari y de Haan, 2013). De los primeros estudios realizados respecto a la mediación y control parental, se obtiene que, entre padres de distintos países y culturas, está generalizado el uso conjunto de internet frente a restricciones de tipo más técnico (bloque de páginas webs) o limitaciones de tiempo (Kirwill, 2009).

En opinión de los propios menores, otras acciones desarrolladas por sus padres son: limitar el tiempo de conexión, revisar el historial, preguntar qué se hace o incluso que el padre o madre se agregue a su red social (García y López de Ayala, 2013). En otro estudio posterior, se encuentra que los menores perciben que existe interés por parte de los padres en conocer qué están haciendo (55\%), seguidos de aquellos que dicen que les revisan con más cercanía para comprobar con quién hablan o por dónde navegan (37.5\%), frente al 30\% que indica que sus padres no hacen nada (30\%) (Moreno, León y Contreras, 2014). Recientes estudios confirman los efectos que determinadas estrategias parentales tienen en la relación de los menores con las TIC, sobre todo en sus actitudes y comportamientos en internet, sirviendo en ocasiones como factores de protección (Hui-Lien, Chien, Chao-Hsiu, 2016). De estas opiniones se desprende que preguntar qué se hace, limitar el tiempo de conexión a internet, revisar el historial o incluso ligar la cuenta de Facebook del hijo /o hija a la propia, no es suficiente para evitar que los menores queden expuestos a riesgos en la red entre los que destaca la ciberadicción, el contacto con desconocidos y el grooming o el cyberbullying.

\section{Objetivos e hipótesis}

Parece pues necesario seguir indagando en la percepción de padres y estudiantes sobre la labor de supervisión familiar para establecer vías de trabajo con ambos colectivos en materia de seguridad del menor en la red. Ante las escasas investigaciones en el contexto de Murcia en este sentido, se analiza el consumo y usos que estudiantes de primaria, secundaria y bachillerato hacen de la telefonía móvil y el ordenador, las formas de supervisión parental en su contexto familiar, y la relación con el uso problemático de las TIC y experiencias de ciberbullying. 


\section{Método}

\section{Participantes}

La muestra, representativa de la Región de Murcia (España), estuvo compuesta por 1914 estudiantes (49.3\% varones, 50.7\% mujeres) con edades comprendidas entre los 11 y los 21 años de edad $(M=13.83$; DT $=2.03)$ de un total de 38 centros educativos. El $61.4 \%$ de los escolares $(n=1175)$ pertenecían a centros públicos y el $36.6 \%$ a centros privados. La distribución por curso fue la siguiente: $6^{\circ}$ curso de primaria $(n=561,29.3 \%)$, Educación Secundaria Obligatoria -ESO- $(n=1169,61.1 \%)$ y Bachillerato $(n=184,9.6 \%)$. La selección muestral fue aleatoria estratificada y por conglomerados. Como unidad primaria de análisis se utilizó el centro educativo elegido al azar por estratos (titularidad del centro) y como unidad secundaria el nivel educativo.

\section{Instrumentos}

La encuesta estructurada autoinformada fue diseñada ad hoc y forma parte de un instrumento más amplio (Giménez, Arnaiz y Maquilón, 2013) que incluye cinco bloques relacionados con: la relación de los menores con las tecnologías (TIC), experiencias de bullying, experiencias de cyberbullying, estrategias de afrontamiento ante el cyberbullying y espectadores del acoso, cuya validez de contenido fue comprobada en varias rondas por juicio de expertos. Para esta investigación se selecionaron únicamente cuatro agrupaciones de preguntas que indagan sobre el acceso y uso de las TIC, implicación en cyberbullying, usos problemáticos de las TIC, supervisión parental y principales estrategias desarrolladas.

Para el primer constructo relacionado con los menores y las TIC, se utilizaron preguntas tipo filtro (“¿tienes teléfono móvil propio?”, “¿utilizas el ordenador o portátil?”) con sí/no como respuesta, seguida de una pregunta de estimación sobre los principales usos del móvil y el ordenador evaluada con una escala tipo likert con cinco opciones de respuesta siendo $1=$ nunca y $5=$ siempre. Algunas opciones ofrecidas fueron: "hacer llamadas", "enviar SMS", "enviar WhatsApp" o "jugar". Para el caso del ordenador, se incluyen ítems referidos a usos académicos ("hacer trabajos del colegio", "buscar información sobre lo que estudio"), usos lúdicos ("jugar") y usos comunicativos ("chatear", "enviar emails", "conectar a redes sociales”). La consistencia interna de esta pregunta fue de $\alpha=.601$.Seguidamente se plantea una 
pregunta de opción múltiple sobre el consumo diario de móvil y ordenador ("menos de 1 hora/día", "entre 1-2 horas/día", "entre 2-4 horas/día" y "más de 4 horas/día").

Para el análisis de las conductas de uso problemático de las TIC se preguntó al alumnado sobre si habían experimentado diversos comportamientos con un total de nueve ítems agrupados en tres categorías conceptuales: a) agresividad ("me enfado cuando me interrumpen y estoy con el móvil o el ordenador", "discuto con algún familiar por estar mucho tiempo con el móvil o el ordenador"); b) cambio de intereses (ej.: "me acuesto tarde por estar con el móvil o en el ordenador", "paso mucho tiempo en mi habitación"); y c) ansiedad (ej.: "me siento mal cuando no puedo utilizar el móvil", "necesito usar mi móvil”). Esta escala se diseñó a partir de la adaptación del cuestionario DENA (Labrador y Villadangos, 2010), obteniéndose valores de fiabilidad de $\alpha=.735$. Para el análisis del cyberbullying se emplearon las dos cuestiones dicotómicas sobre la participación (sí/no) como agresor o como víctima.

La supervisión parental se evaluó a través de una primera pregunta filtro sobre si les vigilaban o controlaban en su acceso a internet o cuando estaban conectados a la red (sí/no). A continuación con dos preguntas de estimación con una escala tipo Likert donde 1 = nunca y 5 = siempre, se les pregunta quién ejerce tal supervisión (ej: "padres/madres", "abuelos", "hermanos mayores" o "profesor") y de qué forma (estrategias) desarrollaban tal supervisión. Algunos ejemplos de estrategias ofrecidas fueron: "me preguntan qué hago, qué veo", "controlan el tiempo que estoy conectado", "controlan qué páginas visito" o "vemos cosas juntos". Esta pregunta presentó una fiabilidad de $\alpha=.746$.

\section{Procedimiento}

Los datos se recogieron entre febrero y junio de 2012 tras solicitar a los centros educativos por contacto telefónico su participación. Recibida la confirmación, se procedió a visitar los centros por parte del investigador principal y la cumplimentación de los cuestionarios por lo estudiantes. Se generó entonces la matriz de datos para su posterior análisis a partir del programa estadístico SPSS versión 21.0.

\section{Análisis de datos}

Se trata de un estudio cuantitativo no experimental y transversal de corte descriptivo y exploratorio. Comprobada la normalidad de la muestra e igualdad de varianzas se optó por la vía paramétrica. En primer lugar, se recategorizaron las variables con escala Likert de cinco 
opciones a tres $(1=$ nunca, $2=$ normalmente, $3=$ casi siempre/siempre $)$ para evitar la dispersión en las respuestas. Se utilizó la Chi cuadrado para comprobar la asociación significativa entre variables nominales y la correlación con la Rho de Spearman $(\rho)$ para las de carácter ordinal (edad y nivel educativo). Para el análisis descriptivo se utilizaron frecuencias y porcentajes. Para el contraste de hipótesis de dos grupos se utilizó la $t$ de Student con la $d$ de Cohen $(d)$ para medir el tamaño del efecto y para el contraste de más de dos grupos la ANOVA de un factor, con la eta cuadrado parcial $\left(\mathrm{y}^{2} \mathrm{p}\right)$. El valor de significación se establece en $p$ $<.05$.

\section{Resultados}

Uso y acceso a las TIC

De los 1707 estudiantes que contestaron esta pregunta, el 89.2\% afirma disponer de un teléfono móvil propio, de los que un $69.4 \%$ tiene acceso a internet. En cuanto al ordenador, el 96.4\% afirma tener ordenador con un $98.9 \%$ que accede a internet a través del mismo. La mayoría de los encuestados dice hacer un consumo bajo de la telefonía móvil entre 1-2h/día (68.9\%), un $16.2 \%$ entre 2-4h/día y un $14.9 \%$ más de 4h/día. En el ordenador, el consumo mayoritario se concentra también entre las 1-2h/día (67.1\%), 2-4h/día (22.5\%) y más de 4h/día (10.4\%). Son las mujeres quienes consumen diariamente más la telefonía móvil tanto en la franja de $2-4 \mathrm{~h} /$ día $($ hombre $=44.6 \%$, mujer $=55.4 \%)$ como en más de $4 \mathrm{~h} /$ día $($ hombre $=$ $30.6 \%$, mujer $=69.4 \%)[t=-6.874, \mathrm{gl}=1705, p<.000, d=0.28]$. Las diferencias no resultan significativas para el caso del consumo del ordenador, aunque ellas vuelven a destacar en el consumo de más de $4 \mathrm{~h} /$ día $(54.7 \%)$ respecto a los varones $(45.3 \%)$.

En cuanto a los usos que los estudiantes realizan de las TIC (Tabla 1), destaca principalmente el uso comunicativo del móvil a través de WhatsApp, llamadas y mensajes de texto (SMS), con diferencias significativas por sexo a favor de las chicas $[t=-3.770, \mathrm{gl}=1705, p<$ $.000, d=0.26]$, mientras que los varones hacen un uso preferente del móvil con una finalidad lúdica $[t=3.347, \mathrm{gl}=1705, p<.001, d=0.20]$. En el caso del ordenador, resalta el uso prioritario para la comunicación interpersonal sobre todo a través de las redes sociales, aunque en este caso las diferencias no resultaron estadísticamente significativas $[t=-1.493, \mathrm{gl}=1844 \mathrm{p}$ $=.136]$. Sí que aparecen diferencias por sexo en el uso lúdico del ordenador, más propio de varones que de mujeres $[t=-10.231, \mathrm{gl}=1844, p<.000, d=0.44]$. Por su parte, son ellas las 
que realizan un uso más académico para realizar tareas escolares o buscar información en comparación con los varones $[t=-6.591, \mathrm{gl}=1844, p<.000, d=0.09]$. Al cruzar este variable con el nivel educativo no se encontraron diferencias en el uso lúdico del teléfono móvil pero sí en los usos comunicativos $\left[F=43.926, \mathrm{gl}=2, p<.000, \mathrm{y}_{\mathrm{p}}^{2}=.05\right]$ más propio este de los estudiantes de bachillerato que los de ESO y primaria. Los usos del ordenador difieren también por nivel educativo tanto en su uso académico más propio de los escolares de primaria $\left[F=9.761, \mathrm{gl}=2, p<.000, \mathrm{y}_{\mathrm{p}}^{2}=.01\right]$, como en su uso lúdico $[F=31.253, \mathrm{gl}=2, p<$ $\left..000, \mathrm{y}_{\mathrm{p}}^{2}=.03\right]$ y comunicativo $\left[F=36.014, \mathrm{gl}=2, p<.000, \mathrm{y}_{\mathrm{p}}^{2}=.04\right]$ más propio de adolescentes de secundaria.

Tabla 1. Resumen de los usos de la telefonía móvil e internet por sexo

\begin{tabular}{|c|c|c|c|c|c|c|}
\hline Teléfono móvil $(n=1707)$ & Sexo & $M(D T)$ & $t(\mathrm{gl})$ & $g l$ & $p$ & $d$ \\
\hline \multirow{2}{*}{ Llamadas } & Hombre & $2.02(0.85)$ & \multirow{2}{*}{-2.22} & \multirow{2}{*}{1705} & \multirow{2}{*}{.026} & \multirow{2}{*}{$\mathrm{ns}$} \\
\hline & Mujer & $2.11(0.83)$ & & & & \\
\hline \multirow{2}{*}{ SMS } & Hombre & $1.43(0.71)$ & \multirow{2}{*}{-7.91} & \multirow{2}{*}{1705} & \multirow{2}{*}{$<.000$} & \multirow{2}{*}{.35} \\
\hline & Mujer & $1.74(0.84)$ & & & & \\
\hline \multirow{2}{*}{ MMS } & Hombre & $1.11(0.41)$ & \multirow{2}{*}{.95} & \multirow{2}{*}{1705} & \multirow{2}{*}{.344} & \multirow{2}{*}{ ns } \\
\hline & Mujer & $1.09(0.38)$ & & & & \\
\hline \multirow{2}{*}{ WhatsApp } & Hombre & $1.80(0.94)$ & \multirow{2}{*}{-.69} & \multirow{2}{*}{1705} & \multirow{2}{*}{.486} & \multirow{2}{*}{ ns } \\
\hline & Mujer & $1.83(0.96)$ & & & & \\
\hline \multirow{2}{*}{ Jugar } & Hombre & $1.71(0.85)$ & \multirow{2}{*}{6.16} & \multirow{2}{*}{1705} & \multirow{2}{*}{$<.000$} & \multirow{2}{*}{.27} \\
\hline & Mujer & $1.47(0.77)$ & & & & \\
\hline \multirow{2}{*}{ Internet } & Hombre & $1.87(0.92)$ & \multirow{2}{*}{-.45} & \multirow{2}{*}{1705} & \multirow{2}{*}{.652} & \multirow{2}{*}{$\mathrm{ns}$} \\
\hline & Mujer & $1.89(0.94)$ & & & & \\
\hline \multirow{2}{*}{ Emails } & Hombre & $1.22(0.56)$ & \multirow{2}{*}{1.84} & & & \\
\hline & Mujer & $1.18(0.52)$ & & $1 / 05$ & .066 & ns \\
\hline Ordenador $(n=1846)$ & & & & & & \\
\hline Tarea & Hombre & $1.67(0.77)$ & & 1814 & & \\
\hline Tarea & Mujer & $1.94(0.85)$ & .27 & 182 & 00 & .31 \\
\hline Búcaueda información & Hombre & $1.49(0.73)$ & 2 & 1844 & 000 & 16 \\
\hline ormacion & Mujer & $1.63(0.81)$ & 2 & 44 & 00 & .10 \\
\hline Jugar & Hombre & $1.94(0.89)$ & & 1844 & 000 & 45 \\
\hline Jugal & Mujer & $1.53(0.81)$ & & 1044 & 000 & .4J \\
\hline Chat & Hombre & $2.13(0.92)$ & & 1844 & & \\
\hline Cnaléc & Mujer & $2.19(0.91)$ & .49 & 1844 & .134 & ns \\
\hline Emails & Hombre & $1.61(0.81)$ & 7 & 1844 & $4 / 6$ & $\mathrm{~ns}$ \\
\hline Emants & Mujer & $1.63(0.84)$ & -.71 & 1044 & $.4 / 0$ & IIS \\
\hline Redes sociales & Hombre & $2.46(0.82)$ & & 1844 & 263 & ns \\
\hline & Mujer & $2.50(0.81)$ & & $10+4$ & & \\
\hline
\end{tabular}




\section{Supervisión parental ante el acceso a las TIC}

Respecto a la supervisión parental durante la conexión a internet, el 69\% ( $n=572)$ de los encuestados declara ser supervisado por algún familiar durante su conexión a internet, concretamente a través del ordenador. Al considerar el sexo, son las mujeres quienes afirman ser más supervisadas que los hombres $[t=-5.229, \mathrm{gl}=1844, p<.000, d=.18]$. Cuando se les pregunta quién ejerce tal supervisión, son ambos progenitores los principales encargados de esta tarea, seguidos de los hermanos mayores. De nuevo son las mujeres las que afirman ser más supervisadas por sus padres que los varones $[t=-1.900, \mathrm{gl}=571, p=.050, d=.16]$, mientras que con el resto de personas que ejercen esta labor no aparecen diferencias por sexo. También aparecen diferencias por edad $\left[F=3.160, \mathrm{gl}=2, p=.043, \mathrm{y}_{\mathrm{p}}^{2}=.01\right]$ y nivel educativo $\left[F=6.435, \mathrm{gl}=2, p=.002, \mathrm{y}_{\mathrm{p}}^{2}=.02\right]$, con correlaciones significativas negativas entre supervisión familiar y la edad $(\rho=-.111, p=.008)$ y supervisión familiar y nivel educativo $(\rho=$ $-.138, p<.001)$.

A continuación, se analizaron las estrategias que, en opinión de los estudiantes, la familia pone en marcha durante su conexión a internet. La forma más común de supervisión es a través de preguntas directas, seguida del control y límite de tiempo y la revisión del historial. Estrategias menos comunes son el "acceso conjunto" o "registrar el ordenador". Únicamente aparecen diferencias significativas por sexo en la estrategia referida a realizar preguntas directas más común hacia las mujeres que hacia los varones $[t=-2.292, \mathrm{gl}=571, p=.022$, $d=.18$ ]. Tal y como se observa en la Tabla 2, aparecen diferencias significativas al considerar el nivel educativo en las variables de registro, control del historial y acceso conjunto, siendo más comunes hacia los hijos de primaria y disminuyendo en ESO y bachillerato.

Tabla 2. Estrategias de supervisión parental por edad y nivel educativo

\begin{tabular}{|c|c|c|c|c|c|c|}
\hline $\begin{array}{l}\text { Estrategias } \\
(n=573)\end{array}$ & Nivel educativo & $M(\mathrm{DT})$ & $F(\mathrm{gl})$ & gl & $p$ & $y_{p}^{2}$ \\
\hline \multirow{3}{*}{ Preguntas } & Primaria & $2.17(0.87)$ & \multirow{3}{*}{1.907} & \multirow{3}{*}{2} & \multirow{3}{*}{.149} & \multirow{3}{*}{ ns } \\
\hline & ESO & $2.08(0.89)$ & & & & \\
\hline & Bachillerato & $2.00(0.92)$ & & & & \\
\hline \multirow{3}{*}{ Límite del tiempo } & Primaria & $2.01(0.93)$ & \multirow{3}{*}{.854} & \multirow{3}{*}{2} & \multirow{3}{*}{.426} & \multirow{3}{*}{ ns } \\
\hline & ESO & $1.92(0.91)$ & & & & \\
\hline & Bachillerato & $1.85(0.82)$ & & & & \\
\hline \multirow{3}{*}{ Registro del ordenador } & Primaria & $1.45(0.79)$ & \multirow{3}{*}{13.611} & \multirow{3}{*}{2} & \multirow{3}{*}{$<.000$} & \multirow{3}{*}{.05} \\
\hline & ESO & $1.19(0.65)$ & & & & \\
\hline & Bachillerato & $1.07(0.53)$ & & & & \\
\hline
\end{tabular}




\begin{tabular}{|c|c|c|c|c|}
\hline \multirow{3}{*}{ Control del historial } & Primaria & $1.77(0.89)$ & \multirow{3}{*}{000} & \multirow{3}{*}{.09} \\
\hline & ESO & $1.29(0.65) 31.090$ & & \\
\hline & Bachillerato & $1.07(0.27)$ & & \\
\hline \multirow{3}{*}{ Acceso conjunto } & Primaria & $1.57(0.82)$ & \multirow{3}{*}{001} & \\
\hline & ESO & $1.33(0.66) \quad 7.670$ & & .03 \\
\hline & Bachillerato & $1.26(0.59)$ & & \\
\hline
\end{tabular}

\section{Supervisión parental y su asociación con riesgos asociados a las TIC}

Analizados el consumo y usos de las TIC y las principales estrategias de supervisión parental, se analiza la posible asociación entre ésta y el consumo de la telefonía móvil y el ordenador y riesgos asociados como el uso problemático o posible ciberadicción, y experiencias de cyberbullying. Para el primer caso, no se encuentra asociación estadísticamente significativa entre la supervisión parental y un mayor o menor consumo de los estudiantes del teléfono móvil. Sin embargo, sí que el control parental está asociado con un mayor consumo del ordenador por parte de los escolares $\left[\chi^{2}=7.99, \mathrm{gl}=2, p=.018 ; \rho=-.054, p=.020\right]$. Respecto al uso problemático y posible ciberadicción, no se encontraron diferencias significativas entre los escolares que afirmaban ser supervisados y los que no lo eran. Por el contrario, cuando se realiza el análisis de acuerdo a cada una de las tres dimensiones o categorías (ansiedad, comportamientos agresivos y de cambio de intereses), se observa que la supervisión parental está asociada de forma significativa con conductas referidas a cambio de intereses $\left[\chi^{2}=18.74, \mathrm{gl}=\right.$ $12, p=.019 ; \rho=-.067, p=.004]$. Los escolares que afirman no ser supervisados muestran una puntuación media significativamente mayor en conductas de cambios de intereses que los que sí son supervisados $[t=2.356, \mathrm{gl}=1844, p=.019)$. Igualmente se encontró asociación estadísticamente significativa entre la supervisión parental y la implicación en dinámicas de ciberbullying $\left[\chi^{2}=31.01, \mathrm{gl}=1, p<.000 ; \rho=.130, p<.000\right]$. El $87.9 \%$ de los escolares que afirman ser supervisados por la familia no están implicados como cibervíctimas. En el caso de la ciberagresión, la asociación con el control parental no resultó significativa $\left[\chi^{2}=3.62\right.$, gl $=$ $1, p=.057 ; \rho=.044, p=.057]$.

\section{Discusión y Conclusiones}

Los resultados respecto a la generalización en el acceso y uso del teléfono móvil y el ordenador por parte de niños y adolescentes, confirman la naturalidad y consumo generalizado de las TIC que ya se ha demostrado en estudios previos en España (Alfaro et al., 2015; 
Berríos et al., 2015; Ministerio del Interior, 2014; ONTSI, 2016). Aunque si bien los escolares de la Región de Murcia afirman realizar un uso mayoritario del móvil y el ordenador entre una y dos horas al día, entre un $10 \%$ y $15 \%$ confirma dedicar más de cuatro horas diarias al móvil y al ordenador, respectivamente. Estos resultados se asemejan a los encontrados por Rial, Gómez, Braña y Varela (2014) en una muestra de adolescentes gallegos. Además, aparecen diferencias significativas por sexo siendo las mujeres quienes hacen un consumo mayor del teléfono móvil, datos coincidentes a los de Malo, Casas, Figuer y González (2006).

En los usos del móvil destacan el WhatsApp, las llamadas y SMS. Son ellas quienes hacen un uso preferentemente comunicativo del mismo mientras que los chicos se decantan por el uso lúdico. En el caso del ordenador, vuelven a aparecer diferencias significativas por sexo siendo ellas las que hacen un uso más académico mientras ellos se decantan por jugar. La herramienta por antonomasia son las redes sociales, dato que se contrapone a los datos reportados en Europa por Ólafsson et al. (2013) que advertían del uso del ordenador para realizar tareas escolares y con finalidad lúdica. La profusión en el uso generalizado de las redes sociales por parte de la población adolescente es una realidad (Ministerio del Interior, 2014) incluso ya en preadolescentes de primaria (Fernández, Peñalva e Irazabal, 2015). MartínezFerrery Moreno (2017) constatan además la relación entre la dependencia a las redes sociales y conductas de violencia relacional escolar entre iguales. Esto nos lleva a reflexionar sobre los peligros a los que se enfrentan los menores durante su tiempo de conexión a la red cuando además la supervisión parental es cuestionable. Parece clara la necesidad de abogar y trabajar por la ciberconvivencia educando a los menores en el uso seguro y responsable de las TIC (Ortega y Zych, 2016), para que eviten, sepan afrontar y denunciar situaciones de ciberacoso o violencia virtual en otros contextos, para hacer del ciberespacio un lugar seguro en el que relacionarse, mantener y hacer nuevas amistades, informase, aprender y comunicarse de forma positiva.

La implicación de la familia es, en todo caso, clave y determinante. Contemplar este escenario desde la responsabilidad exclusiva de la escuela como entorno de aprendizaje y convivencia supone una visión estrecha y mezquina de un proceso que ha de asentar sus bases en los modelos educativos de la escuela y la familia. El riesgo en la ciberconducta es una coyuntura explícita. Una aventura cargada de incertidumbres en la que los adultos hemos de estar de modo explícito y acompañar a los menores porque "educar no es, no puede ser, asus- 
tar. Sino ilustrar, acompañar, hacer juntos, opinar, mediar, explicar, medir, reflexionar (...)" (Luengo, 2014, 8). Se trata de generar espacios y momentos de reflexión con los menores a través de la mediación parental y del profesorado en los tiempos de uso de las TIC en pro del aprendizaje de la ciudadanía digital sana y responsable. Investigaciones recientes constatan que la mediación parental disminuye la probabilidad de que los menores se vean inmersos en experiencias de cyberbullying (Navarro, Serna, Martínez y Ruiz-Oliva, 2013), incluso a traés de herramientas concretas como Facebook (Saunders y Varma, 2015), a la par que los empodera para su uso responsable y aprovechar las oportunidades que ofrece internet (Blinka, 2013).

Nuestros resultados van en esta línea encontrándose relación significativa entre la escasez de supervisión familiar y la implicación en dinámicas de ciberacoso, como también con el uso problemático y de posible ciberaddición y un consumo excesivo del ordenador. Autores como Blinka (2013) y Kalmus, Blinka y Ólafsson (2015) concluyen que el control parental, incluso de tipo restrictivo en cuanto al tiempo de conexión, reduce el consumo excesivo de internet y las TIC. Resultados similares son los obtenidos por Saunders y Varma (2015) que encuentran que una supervisión parental a través de aconsejar y guiar a los hijos, reduce el tiempo de conexiónn a Facebook, como también las posibilidades de ser victimizado a través de esta herramienta. En nuestro caso, se observa que los menores que dicen ser menos supervisados presentan más conductas de cambio de intereses (aislamiento en la habitación para estar con el móvil, ordenador o videojuegos, preferir estar con las TIC que hacer deporte o estar con amigos), que aquellos otros que sí son más supervisados.

A ello se suma la realidad de que siete de cada diez alumnos advierte no ser supervisado durante su conexión a la red, y cuando sí lo son, son las hijas las más controladas en comparación con los hijos, datos similares a los obtenidos por Garmendia et al. (2013). Esto se suma a resultados internacionales que apuntan el escaso conocimiento que las familias tienen sobre los riesgos en internet y las actividades que realizan sus hijos en la red (Symons, Ponnet, Emmery, Walrave y Heirman, 2017). La edad también parece ser un factor condicionante en la supervisión parental descendiendo a la par que los niños pasan a ser adolescentes, constatado también en estudios previos (Berríos et al., 2015). Esto es un dato curioso coincidente con el hecho de que en la adolescencia el grupo de iguales es el que se convierte en el principal referente y apoyo en las actividades y peligros existentes en la red (Jiménez, Garmendia y Casado, 2015). 
La supervisión que los menores encuestados afirman recibir es principalmente activa y directa a través de preguntas sobre qué se hace en internet, con quién se habla, etc., seguida de la restricción y control del tiempo de conexión. A pesar de que el acceso conjunto en internet es una buena estrategia de mediación y que permite un intercambio comunicativo y de relación entre padres e hijos, es de las menos referidas por parte de los adolescentes, hecho contrario a lo reportado por Symons, Ponnet, Walrave y Heirman (2017) en un acercamiento cualitativo a la cuestión en la que concluyen que la comunicación abierta con los hijos y la conexión conjunta son las estrategias preferidas en término de mediación parental. El sexo y el nivel educativo están asociados de forma significativa al desarrollo de unas u otras estrategias de mediación. Según los escolares, son las mujeres quienes dicen ser más preguntadas y más los escolares de primaria a los que se les registra más el ordenador, controla el historial y se comparte tiempo de acceso conjunto frente a los adolescentes de la ESO y bachillerato.

Esta investigación presenta varias limitaciones referidas al instrumento de recogida de información. La extracción de varias preguntas con estructura y opciones de respuesta diversa dificulta el análisis factorial, como también la extensión de la pregunta referida a las estrategias de supervisión parental. Futuras investigaciones deberán partir de los avances actuales en materia de supervisión parental e instrumentos más estandarizados para tener una visión más amplia de esta realidad en el contexto en que se enmarca esta investigación de aproximación inicial al análisis del control parental y exposición a riesgos entre menores de Murcia (España). Igualmente sería interesante comparar la visión de padres e hijos sobre la supervisión parental, incidiendo expresamente en el fenómeno del sexting. Conocer los patrones de uso de las tecnologías, la percepción que los menores tienen de la supervisión parental y su asociación con riesgos en la red constata la necesidad de seguir trabajando activamente en el diseño y desarrollo en la escuela y la familia de estrategias de respuesta, afrontamiento y superación ante situaciones que acechan la integridad y desarrollo de posibles víctimas con consecuencias notables en la configuración y despliegue de la personalidad, autoconcepto, autoestima, relaciones interpersonales y seguridad afectiva de niños y adolescentes. 


\section{Referencias}

Alfaro, M., Vázquez, M. E., Fierro, A., Herrero, B., Muñoz, M. F., Rodríguez, L., y Grupo de Educación para la Salud de la AEPap (2015). Uso y riesgos de las tecnologías de la información y la comunicación en adolescentes de 13-18 años. Acta Pediátrica Española, 73(6), 146-151.

Bellamy, A., y Yousef, W. (2015). The impact of cyberbullying on the self-esteem and academic performance of Arab-American high school students. Electronic Journal of Research in Educational Psychology, 13(3), 463-482. doi: 10.1420/ejrep.37.15011

Berríos, L., Buxarrais, M. R., y Garcés, M. S. (2015). Uso de las TIC y mediación parental percibida por niños de Chile. Comunicar, 45(XXIII), 162-168. doi: 10.3916/C45-201517

Blinka, L. (2013). The "good" and the "bad" of the internet: Studying subjective well-being as an outcome of young people's online practices (Thesis Dissertation). University of Tartu, Estonia.

Calmaestra, J., Escorial, A., García, P., del Moral, C., Perazzo, C., y Ubrich, T. (2016). Yo a eso no juego. Bullying y ciberbullying en la infancia. Madrid: Save The Children.

Carbonell, X., Fúster, H., Chamarro, A., y Oberst, U. (2012). Adicción a internet y móvil: Una revisión de estudios empíricos españoles. Papeles del Psicólogo, 33(2), 82-99.

Cloquell, A. (2015). Usos sociales de internet entre los adolescentes españoles. Revista sobre la infancia y la adolescencia, 8, 1-14. doi: 10.4995/reinad.2015.3649

Colás, P., González, T., y de Pablos, J. (2013). Juventud y redes sociales: Motivaciones y usos preferentes. Comunicar, 40(XX), 15-23. doi: 10.3916/C40-2013-02-01.

Dalla, V., Di Pietro, A., Morel, S., y Psaila, E. (2016). Cyberbullying among young people. London: Policy Department for Citizen's Rights and Constitutional Affairs.

Del Río, Sádaba, C. y Bringué, X. (2010). Menores y redes ¿sociales?: de la amistad al cyberbullying. Revista de Estudios de Juventud, 88, 115-129.

Fajardo, M. I., Gordillo, M., y Regalado, A. B. (2013). Sexting: Nuevos usos de la tecnología y la sexualidad en adolescentes. International Journal of Developmental and Educational Psychology, 1, 521-533.

Fernández, J., Peñalva, A., e Irazabal, I. (2015). Hábitos de uso y conductas de riesgo en internet en la preadolescencia. Comunicar, 44(XXII), 113-120. doi: 10.3916/C44-201512 
Finkelhor, D., Mitchell, K. J., y Wolak, J. (2000). Online victimization: A report on the nation's youth. Alexandria, VA: National Center for Missing and Exploited Children.

Fu, K.-W., Chan, C.-C., y Ip, P. (2014). Exploring the relationship between cyberbullying and unnatural child death: an ecological study of twenty-four European countries. $B M C$ Pediatrics, 14, 195-200. doi: 10.1186/1471-2431-14-195

García, B. C., y López de Ayala, M. C. (2013). Los padres ante el uso de internet y redes sociales por menores. Control y protección. Comunicación presentada al V Congreso Internacional Latina de Comunicación Social, Universidad de La Laguna.

Giménez, A. M., Arnaiz, P. y Maquilón, J. J. (2013). CYBERBULL Questionnaire [Database record]. APA PsychTest. doi: 10.1037/t40201-000

Giménez, A. M., Hunter. S. C., Durking, K., Arnaiz, P., y Maquilón, J. J. (2015). The emotional impact of cyberbullying: Differences in perceptions and experiences as a function of role. Computers \& Education, 82, 228-235. doi: 10.1016/j.compedu.2014.11.013

Garmendia, M., Casado, M. A., Martínez, G., y Garitaonandia, C. (2013). Las madres y padres, los menores e internet. Estrategias de mediación parental en España. Doxa Comunicación, 17, 99-117.

Hase, C. N., Goldberg, S. B., Smith, D., Stuck, A., y Campain, J. (2015). Impacts of traditional bullying and cyberbullying on the mental health of middle school and high school students. Psychology in the Schools, 52(6), 607-617. doi: 10.1002/pits.21841

Helsper, E. J., Kalmus, V., Hasebrink, U., Sagvari, B., y de Haan, J. (2013). Country classification: opportunities, risks, harm and parental mediation. London: EU Kids Online, The London School of Economics and Political Science.

Hinduja, S., y Patchin, J. W. (2015). 2015 Cyberbullying Data. Recuperado de http://cyberbullying.org/2015-data

Hui-Lien, C., Chien, C., Chao-Hsiu, C. (2016). The moderating effects of parenting styles on the relation between the internet attitudes and internet behaviors of high-school students in Taiwan. Computers \& Education, 94, 204-214. doi: 10.1016/j.compedu.2015.11.017

Jiménez, E., Garmendia, E., y Casado, M. A. (2015). Percepción de los y las menores de la mediación parental respecto a los riesgos en internet. Revista Latina de Comunicación Social, 70, 49-68. doi: 10.4185/RLCS-2015-1034 
Kalmus, V., Blinka, L., y Ólafsson, K. (2015). Does it matter what mama says: Evaluating the role of parental mediation in European adolescents' excessive internet use. Children \& Society, 29(2), 122-133. doi: 10.1111/chso.12020

Kirwill, L. (2009). Parental mediation of children's internet use in diferent european countries. Journal of Children and Media, 3(4), 394-409. doi: 10.1080/17482790903233440

Kowalski, R. M., Giumetti, G. W., Schroeder, A. N., y Lattanner, M. R. (2014). Bullying in the digital age: a critical review and meta-analysis of cyberbullying research among youth. Psychological Bulletin, 140(4), 1073-1137. doi: 10.1037/a0035618

León, B., Felipe, E., Fajardo, F., y Gómez, T. (2012). Cyberbullying en una muestra de estudiantes de educación secundaria: Variables moduladoras y redes sociales. Electronic Journal of Research in Educational Psychology, 10(29, 771-788.

Lucas, B., Pérez, A., y Giménez, M. (2016). La evaluación del ciberbullying: Situación actual y retos futuros. Papeles del psicólogo, 37(1), 27-35.

Luengo, J. A. (2014). Ciberbullying, prevenir y actuar. Madrid: Colegio Oficial de Psicólogos de Madrid y Fundación A3Media.

Malo, S., Casas, F., Figuer, C., y González, M. (2006). El teléfono móvil: disponibilidad, usos y relaciones por parte de los adolescentes entre 12 y 16 años. Estudios sobre Educación, 10, 55-78.

Martínez-Ferrer, B. y Moreno, D. (2017). Dependencia de las redes sociales virtuales y violencia escolar en adolescentes. International Journal of Developmental and Educational Psychology, 2(1), 105-114. doi: 10.17060/ijodaep.2017.n1.v2.923

Martínez de Morentin, J. I., y Medrano, C. (2012). La mediación parental y el uso de internet. International Journal of Developmental and Educational Psychology, 1(1), 549-556.

Ministerio del Interior (2014). Encuesta sobre hábitos de uso y seguridad de internet de menores y jóvenes en España. Recuperado de http://www.interior.gob.es/prensa/noticias//asset_publisher/GHU8Ap6ztgsg/content/id/2735634

Mishna, F., McInroy, L., Lacombe-Duncan, A., y Daciuk, J. (2015). Motivations for cyberbullying: A longitudinal and multi-perspective inquiry. Toronto: University of Toronto.

Monks, C. P., Mahdavi, J., y Rix, K. (2016). The emergence of cyberbullying in childhood: Parent and teacher perspectives. Psicología Educativa, 22,39-48. doi: 10.1016/j.pse.2016.02.002

Moreno, D. E., León, G. A., y Contreras, C. R. (2014). El uso seguro de internet y el rol de los padres. Revista Internacional de Tecnologías en la Educación, 1(1), 47-56. 
Muñoz, R., Ortega, R., López, M. R., Batalla, C., Manresa, J. M., Montellá, N., y Torán, P. (2016). The problematic use of Information and Communication Technologuies (ICT) in adolescents by the cross sectional JOITIC study. BMC Pediatrics, 14, 140-151. doi: 10.1186/s12887-016-0674-y

Navarro, R. y Serna, C. (2016). Spanish youth perceptions about cyberbullying: Qualitative research into understanding cyberbullying and the role that parents play in its solution. En R. Navarro, S. Yubero y E. Larrañaga (eds.), Cyberbullying across the globe. Gender, family, and mental Health (pp. 193-218). Switzerland: Springer.

Navarro, R., Serna, C., Martínez, V., y Ruiz-Oliva, R. (2013). The role of internet use and parental mediation on cyberbullying victimization among Spanish children from rural public schools. European Journal of Psychology of Educativon, 28(3), 727-745. doi: $10.1007 / \mathrm{s} 10212-012-0137-2$

Ochaita, E., Espinosa, M. A., y Gutiérrez, H. (2011). Las necesidades adolescentes y las nuevas tecnologías de la información y la comunicación. Revista de Estudios de Juventud, $92,87-110$.

Observatorio Nacional de las Telecomunicaciones y de la SI (2016). Indicadores sobre uso de TIC por menores en España. Madrid: Ministerio de Energía, Turismo y Agenda Digital.

Ortega, R. y Zynch, I. (2016). La ciberconducta y la psicología educativa: retos y riesgos. Psicología Educativa, 22(1), 1-4. doi: 10.1016/j.pse.2016.04.001

Rial, A., Gómez, P., Braña, T., y Varela, J. (2014). Percepciones y uso de internet y las redes sociales entre los adolescentes de la comunidad gallega (España). Anales de Psicología, 30(2), 642-655. doi: 10.6018/analesps.30.2.159111

Sakellariou, T., Carroll, A., y Houghton, S. (2012). Rates of cyber victimization and bullying among male Australian primary and high school students. School Psychology International, 33(5), 533-549. doi: 10.1177/0143034311430374

Saunders, C., y Varma, P. (2015). The relationship between parental mediation and facebook victimization and in-person victimization, both directly and indirectly being mediated by the intesity of facebook use by middle school students in an international school in Bangkok, Thailand. Scholar, 8(1), 201-213.

Smith, P. K. (2016). Foreword: An international perspective on cyberbullying. En R. Navarro, S. Yubero y E. Larrañaga (eds.), Cyberbullying across the globe. Gender, family, and mental Health (pp. v-vi). Switzerland: Springer. 
Symons, K., Ponnet, K., Emmery, K., Walrave, M. y Heirman, W. (2017). Parental knowledge of adolescents' online content and contact risks. Journal of Youth and Adolescence, 46, 401-416. doi: 10.1007/s10964-016-0599-7

Symons, K., Ponnet, K., Walrave, M. y Heirman, W. (2017). A qualitative study into parental mediation of adolescents' internet use. Computers in human behaviour, 73, 423-432. doi: 10.1016/j.chb.2017.04.004 